\title{
Un modelo multiculturalista más allá de la tolerancia
}

\author{
LEÓN OLIVÉ \\ Instituto de Investigaciones Filosóficas \\ Universidad Nacional Autónoma de México \\ olive@servidor.unam.mx
}

\begin{abstract}
Resumen: En este artículo defiendo la tesis de que en las sociedades multiculturales donde hay desigualdades económicas, sociales y materiales entre pueblos o entre culturas, el establecimiento por parte del Estado, o de entidades supraestatales o de organismos internacionales de políticas de compensación o de un trato diferencial en la asignación de recursos a favor de los pueblos o de las culturas que tienen desventajas, se justifica sobre la base de un principio de justicia social. Se discute la noción pertinente de justicia social, basada en la idea de la satisfacción de las necesidades básicas de todos los miembros de la sociedad.
\end{abstract}

Palabras clave: multiculturalismo, justicia social, obligaciones del Estado, necesidades básicas

En este artículo defenderé la tesis de que en las sociedades multiculturales donde hay desigualdades entre pueblos o entre culturas, el establecimiento por parte del Estado, o de entidades supraestatales, o de organismos internacionales, de políticas de compensación o de un trato diferencial en la asignación de recursos a favor de los pueblos o de las culturas que tienen desventajas, se justifica sobre la base de un principio de justicia social. ${ }^{1}$

Una primera y tentativa formulación del principio de justicia pertinente es la siguiente:

Una condición necesaria para que una sociedad sea justa es que establezca los mecanismos que garanticen las condiciones y la distribu-

\footnotetext{
${ }^{1}$ Llamo "multicultural" a una sociedad en la que conviven pueblos diversos o comunidades significativas que se identifican con algún pueblo. Utilizo generalmente los conceptos de "pueblo" y de "cultura" como sinónimos, significando una comunidad de seres humanos que tienen una tradición cultivada a lo largo de varias generaciones, que comparten una o más lenguas, una historia, valores, creencias, instituciones y prácticas (educativas, religiosas, económicas, tecnológicas, etc.), que mantienen expectativas comunes y se proponen desarrollar un proyecto común (cfr. Kymlicka 1995, pp. 18 y 76; Salmerón 1998; Villoro, 1998, pp. 109 y ss.). Éste es el concepto que ha permitido una gran cantidad de trabajo antropológico y que tiene referentes de los que no podemos dudar cuando se habla, por ejemplo, de las culturas (o pueblos) nahua, maya, tzotzil, huichola, tojolabal, judía o palestina. No obstante, en ocasiones por cultura me refiero a un grupo social que se identifica con un pueblo, aunque no sea idéntico a él. Por ejemplo, los judíos en España, o los mexicanos en Estados Unidos. El sentido en que se usa cada término quedará claro en el contexto.
} 
ción de bienes de modo que se satisfagan las necesidades básicas de todos sus miembros.

Más adelante discutiremos la noción de "necesidades básicas" como aquellas cuya satisfacción es indispensable para la realización de un plan de vida. Por ahora podemos subrayar que para muchos individuos que han crecido en el seno de un pueblo o de una cultura, la realización de su plan de vida depende de la existencia y de la preservación, o incluso del florecimiento y el desarrollo, de su cultura. Sobre estas premisas sostendremos que la preservación de una cultura, e incluso el establecimiento de políticas diferenciales a favor de las culturas o de los pueblos con desventajas, es una cuestión de justicia social.

Una consecuencia de esta tesis es que un Estado democrático que asuma la responsabilidad de organizar una sociedad justa tiene la obligación de promover y vigilar que los miembros de las diferentes culturas se toleren unos a otros, estén o no en relaciones de supraordinación y subordinación (tolerancia vertical y tolerancia horizontal, Garzón Valdés 2000, p. 185). Pero bajo esta tesis, el Estado democrático no debe ser el instrumento de ningún pueblo o cultura, y debe mantener una neutralidad frente a todos los pueblos y culturas que forman parte de él. Por esto, de acuerdo con la tesis que fundamentaremos más adelante, sería un error exigir que el Estado democrático tolere a sus ciudadanos, o a los pueblos o culturas a los que ellos pertenecen y que forman parte de él. ${ }^{2}$

Para sustentar lo anterior, analizaremos en primer lugar el concepto de necesidad; en segundo lugar, la forma que debería tomar el principio de justicia en términos de satisfacción de necesidades básicas dentro de una sociedad multicultural, que sea útil para el establecimiento de políticas sociales; y en tercer lugar, las responsabilidades del Estado en una sociedad democrática multicultural justa.

\section{Justicia y necesidad}

Un principio de justicia entendido como satisfacción de necesidades básicas es compatible y debe complementarse con un principio de igualdad

${ }^{2}$ Agradezco a la dictaminadora anónima de Diánoia la observación que me ha permitido hacer estas precisiones.

También debo aclarar que si bien se deriva de la tesis central de este trabajo la obligación del Estado de mantenerse neutral con respecto a los pueblos que forman parte de él, no se trata de la "neutralidad" que sostienen algunas teorías liberales, cuya fundamentación es muy discutible, y que de hecho he discutido en mi libro Multiculturalismo y pluralismo (Olivé 1999). Considero que el argumento que desarrollo a continuación fundamenta tanto la obligación del Estado de establecer políticas compensatorias para los pueblos en desventaja, como la obligación de mantener una posición neutral frente a los pueblos que forman parte de él, en el sentido de que el Estado no debe ser controlado por ninguno de ellos en particular, sino por instancias que garanticen la participación plural de todos los pueblos involucrados. 
que dice que toda persona debería disfrutar del mismo nivel de bienestar. David Miller, por ejemplo, en un libro sobre Justicia social de 1976 sostenía: "La igualdad se logra dando prioridad a la satisfacción de las necesidades, y luego satisfaciendo la mayor proporción posible de los deseos de cada persona, en la medida que lo permitan los recursos" (Miller 1976, pp. 143144).

En una línea parecida, Ernesto Garzón Valdés ha propuesto un "principio de homogeneización": "Una sociedad es homogénea cuando todos sus miembros gozan de los derechos directamente vinculados con la satisfacción de sus necesidades básicas" (Garzón Valdés 1993c, p. 45).

Una formulación alternativa la ofrece el mismo Garzón Valdés discutiendo las muy sugerentes ideas de Mario Bunge sobre necesidades básicas: "Una sociedad es homogénea si y sólo si todos sus miembros tienen normalmente la posibilidad de satisfacer sus necesidades básicas [...] y gozan del respeto de sus deseos legítimos" (Garzón Valdés 1993b, p. 431).

En la primera versión, Garzón Valdés alude, como en muchas teorías de la justicia, a la garantía de que todos los individuos de una sociedad gocen de los derechos que les permitirían satisfacer sus necesidades básicas. Por mi parte prefiero la segunda formulación, que liga la justicia directamente con la posibilidad, normalmente, de satisfacer las necesidades básicas. Se evita así el posible conflicto de llamar justa a una sociedad en la cual se reconoce el derecho de todos a la satisfacción de sus necesidades básicas, pero donde en la práctica no se satisfacen.

\section{Necesidades básicas}

David Miller (1976, p. 127) distingue tres tipos de necesidades:

(1) Necesidades instrumentales ( $A$ necesita un coche para ir de la Ciudad de México a Morelia).

(2) Necesidades funcionales (los profesores necesitan libros y bibliotecas, los cirujanos necesitan habilidades manuales).

(3) Necesidades intrínsecas (las personas necesitan alimento, abrigo, salud).

La categoría de las necesidades instrumentales presupone que los agentes tienen ciertos fines (llegar a Morelia), la obtención de los cuales requiere ciertos medios (un coche, o un boleto de autobús). En esa medida los medios se vuelven necesidades.

En estos casos, la necesidad existe sólo en función del fin propuesto, que es independiente de esa necesidad. Por eso, entender la necesidad requiere conocer el fin. Brian Barry tenía razón, entonces, cuando proponía que 
los enunciados que se refieren a necesidades (de este tipo) son realmente de la forma: " $A$ necesita $X$ para hacer o para lograr $Y$ ". Pero esto es así únicamente para las necesidades instrumentales, no para las funcionales y menos para las necesidades intrínsecas.

En efecto, con respecto a las necesidades funcionales, por ejemplo, para comprender por qué un cirujano necesita habilidad manual, digamos un pulso preciso, o que un profesor requiere libros y revistas, no necesitamos conocer ningún fin adicional que se fije el cirujano o el profesor; sus necesidades no provienen de fines ulteriores, sino de la naturaleza de su profesión. No es que sea absurdo que alguien pregunte por qué un cirujano necesita un pulso preciso, o por qué un profesor requiere libros; pero si alguno lo hace, es porque no comprende qué significa ser un cirujano o un profesor (aunque esto último sea frecuente, por lo menos entre muchas de las autoridades educativas de México).

Con respecto a las necesidades intrínsecas, David Miller sugiere que un significado legítimo - y el que interesa para nuestros propósitos - de la proposición " $A$ necesita intrínsecamente $X$ " es " $A$ sufrirá un daño si carece de X" (Miller 1976, p. 130).

Se trata de necesidades que no provienen de ciertos fines ulteriores que persigue el agente, ni provienen de la naturaleza misma de la profesión o de las circunstancias del agente, sino que son parte misma de los fines. Como dice Miller, si uno se propusiera analizar la situación desvelando el fin que se persigue, simplemente estaría señalando el todo, del cual lo que se necesita es una parte (Miller 1976, p. 128).

Compárense los dos enunciados siguientes:

1. "A necesita un coche (para ir de la Ciudad de México a Morelia)".

2. "A necesita alguien que lo comprenda".

En el primer caso, obviamente se trata de una necesidad que proviene del fin deseado. La necesidad surge porque se requiere un medio para alcanzar el fin. El medio se vuelve entonces necesario. En el segundo caso - aunque podemos decir que $A$ necesita alguien que lo comprenda para estar bien-, tener alguien que lo comprenda, y tenerlo cerca por lo menos en ocasiones importantes para $A$, es parte de lo que significa para $A$ estar bien. La persona comprensiva con $A$ no es sólo un medio para estar bien, es parte del fin de $A$ de estar bien. Si $A$ no tiene nadie que lo comprenda, $A$ no estará bien, y sufrirá un daño. De ahí la propuesta de que " $A$ necesita intrínsecamente $X$ " equivale a " $A$ sufrirá un daño si carece de $X$ " (Miller 1976, p. 130).

"Las personas necesitan intrínsecamente alimento" equivale, entonces, a "si las personas carecen de alimento, sufrirán un daño". Lo mismo pasa con las necesidades intrínsecas de abrigo, de afecto, de comprensión. Pero 
si hacemos descansar el concepto de necesidad intrínseca en el de daño, tenemos ahora que elucidar esta última noción.

\section{Daño}

El concepto de daño aplicado a seres humanos no puede hacerse exclusivamente sobre la base de criterios observacionales, como cuando se habla de daños causados por un incendio en un bosque. Al hablar de daños causados a personas debe considerarse no sólo el nivel físico observable, sino también deben tomarse en cuenta factores subjetivos de la persona: sus proyectos de vida, sus valores, sus aspiraciones y sus ideales (Miller 1976, pp. 132 y ss.).

Éste es uno de los puntos cruciales en las sociedades donde hay diversidad cultural: no hay un único punto de vista correcto acerca de cómo debería vivir la gente ni del tipo de satisfacciones que deberían tener. No hay un único conjunto de criterios de lo que significa una vida buena.

Para determinar qué cuenta como daño para una persona es necesario identificar las metas, los valores y las actividades centrales en la forma de vida de esa persona. Esas metas, valores y actividades son las que constituyen "un plan de vida". Parte de la identidad personal descansa sobre las metas y actividades que constituyen un plan de vida, pues, normalmente, la respuesta a la pregunta "¿quién es usted?" incluye una ubicación social, una referencia - al menos parcial - a ciertas relaciones sociales, así como a actividades que uno normalmente realiza y metas a las que aspira, es decir, la respuesta a la pregunta "¿quién es usted?" incluye una referencia al plan de vida que uno tiene trazado, o al que aspira. Por eso, a veces los daños que puede sufrir una persona afectan incluso las condiciones sobre las que descansa su identidad.

Los planes de vida son múltiples y pueden tomar muy variadas formas. Por ejemplo, desempeñar cierto papel social, público o familiar; perseguir ciertos ideales; hacerse famoso; tener mucho dinero; tener mucho poder y obstaculizar al máximo el trabajo de sus colegas; ayudar a los pobres; incidir en cambios sociales; desarrollarse profesionalmente, como médico, abogado, profesor, científico, filósofo, artista; ser una seductora, etcétera.

La vida de los seres humanos necesariamente incluye un plan en este sentido, así sea el de renunciar a todo y no proponerse nada que vaya más allá de las acciones y fines inmediatos, como algunos vagos que deambulan por las ciudades o por las carreteras modernas.

En las sociedades liberales modernas se ha desarrollado la idea de que las personas tienen el derecho a elegir su plan de vida a partir de un horizonte de posibilidades. En las sociedades tradicionales, por lo general el plan de vida es impuesto a sus miembros y no se espera que ellos lo analicen y que posteriormente lo acepten o lo rechacen racionalmente. Más 
aún, normalmente en una sociedad tradicional las personas no podrían formular explícitamente su plan de vida, pero eso no significa que no lo tengan.

Miller (1976, p. 133) subraya que dado el plan de vida de una persona, sus metas, valores y actividades pueden considerarse dentro de dos grupos: aquéllas que son esenciales para la realización del plan de vida, y aquéllas que no lo son. Las esenciales a su vez incluyen:

a) las que forman parte del plan de vida mismo (e.g., realizar obras de caridad, hacer investigación científica o creación artística, seducir al que se ponga enfrente, enseñar en la escuela primaria, etc.), y

b) aquellas sin cuya satisfacción sería imposible realizar las acciones que constituyen el plan de vida mismo (por ejemplo, tener una alimentación razonable, un estado de salud bueno, etcétera).

A veces las segundas forman parte del primer conjunto también. Por ejemplo, parte del plan de vida de mucha gente, especialmente en las sociedades culturalmente más sofisticadas (tradicionales o modernas), es tener una alimentación que no sólo permita la sobrevivencia, sino que signifique la satisfacción de los sentidos, la obtención de placer físico y psicológico, la satisfacción de estándares estéticos (visuales, olfativos, gustativos, táctiles y auditivos), así como el compartir experiencias agradables con otros. Es decir, comer bien - no sólo en el sentido de que la comida sea nutritiva, sino en el de buena cocina- forma parte del plan de vida de mucha gente en las sociedades culturalmente refinadas (como en muchas de la India, de China, de América Latina y de Europa continental).

Las necesidades básicas de una persona, entonces, pueden entenderse como aquéllas que son indispensables para mantener sus capacidades y la posibilidad de que realice las actividades esenciales de su plan de vida.

En consecuencia, podemos entender el daño para una persona como aquello que directa o indirectamente interfiere con su capacidad para desarrollar las actividades esenciales para realizar su plan de vida, o que interfiere con las actividades mismas que constituyen ese plan de vida.

Para decidir cuáles son las necesidades básicas de una persona debemos, primero, identificar su plan de vida; luego, establecer cuáles son las actividades esenciales para ese plan y, por consiguiente, cuáles capacidades son indispensables, y finalmente investigar las condiciones que permiten la realización de esas actividades (Miller 1976, p. 134).

Estas ideas nos permiten distinguir entre necesidades y deseos. Una persona puede desear algo que no necesita básicamente, es decir, aquello que es deseado no es necesario para la realización del plan de vida de esa persona. Incluso es inteligible, desde esta perspectiva, que una persona no sepa qué necesita, pues puede desconocer cuáles son todas las condiciones 
necesarias para la realización de su plan de vida (por ejemplo, la cantidad mínima de proteínas que debería ingerir cotidianamente para mantener una buena salud).

La realización de los planes de vida de muchas personas que han nacido o han crecido en el contexto de ciertas culturas requieren la preservación y el florecimiento de su cultura, pues muchas de sus metas y de sus acciones sólo son posibles y tienen sentido dentro de su contexto cultural. Por esto, las necesidades básicas de personas que pertenecen a diferentes culturas pueden ser distintas. Son muy diferentes las necesidades de un tojolabal en Chiapas que de un profesor universitario en la Ciudad de México o en Madrid.

\section{Justicia social}

¿Por qué deberíamos establecer mecanismos que garanticen la satisfacción de las necesidades básicas de las personas? Puede haber diferentes respuestas a esta pregunta desde un punto de vista moral: deberíamos buscar la satisfacción de las necesidades básicas de las personas, por ejemplo, por humanidad, por generosidad, por benevolencia o por caridad. Pero por ninguna de estas vías llegaríamos a la conclusión de la necesidad de establecer instituciones sociales que garanticen la satisfacción de las necesidades básicas de todos los miembros de una sociedad como una cuestión de justicia. Es decir, ni la humanidad, ni la generosidad ni la caridad sirven para fundamentar una sociedad justa, aunque sí pueden llevarnos a aliviar el sufrimiento de algunos seres humanos y de algunos animales.

Si formulamos de manera más precisa la pregunta: ¿por qué debería una sociedad establecer mecanismos que garanticen la satisfacción de las necesidades básicas de todos sus miembros?, la respuesta sería: debe hacerlo en aras de la justicia social. La idea quizá quede más clara si nos preguntamos por qué un país con un "Estado de bienestar" o con un buen sistema de seguridad social es más justo que uno que no lo tenga.

La tesis es, pues, que una sociedad es justa si tiene mecanismos e instituciones que garanticen la satisfacción de las necesidades básicas de todos sus miembros. ¿Por qué es un asunto de justicia? Simplemente porque la pregunta tiene sentido con base en otra que está presupuesta: ¿qué organización social debería haber para distribuir los recursos de una manera justa?

El concepto de justicia presupuesto aquí se refiere a estados de cosas o situaciones en las que se encuentran seres racionales (por lo tanto con conciencia) y que los afectan, donde por lo menos alguno de ellos puede sufrir una carga o un daño, o disfrutar de algún beneficio. El estado de cosas, o la situación, debe ser susceptible de cambiar por medio de acciones intencionales de seres racionales. El concepto de justicia se refiere, 
entonces, a las formas de distribución de beneficios y de cargas o daños entre seres racionales.

La justicia social se refiere a la distribución de beneficios y de cargas en una sociedad de seres racionales. Una sociedad justa es una sociedad que ha establecido instituciones, mecanismos y organizaciones públicas para distribuir beneficios y cargas, ventajas y desventajas, de una manera que calificamos justa según cierto criterio. El problema, entonces, es cuál criterio aplicar para calificar de justa una manera de hacer esa distribución. El principio de justicia al que me referí desde el principio se basa en el criterio de la garantía de la satisfacción de las necesidades básicas de todos los miembros de la sociedad.

\section{Necesidades básicas legítimas}

Pero ¿qué ocurre cuando, por ejemplo, el plan de vida de una persona es ser piromaniaco, o estrangulador, o ladrón? ¿Es aceptable, y puede de hecho una sociedad organizarse de tal manera que se garantice la satisfacción de todas las necesidades básicas de sus miembros, incluyendo las del piromaniaco?

Para decidir sobre esta cuestión debemos examinar la parte valorativa que necesariamente está incluida en la noción de "necesidad básica", ligada al concepto de "plan de vida". Esto nos llevará a introducir el calificativo de "legítimas" a las necesidades básicas a las que debe aplicarse el criterio de justicia social.

Las necesidades básicas de una persona — hemos visto- son aquellas cuya satisfacción es indispensable para la realización de su plan de vida. Esto puede determinarse sólo si el plan de vida es inteligible, en el sentido de poderse expresar explícita y coherentemente en un lenguaje, pero también en el sentido de que pueda comprenderse por qué, para la persona que tiene ese plan de vida, el mismo tiene significado y valor (Miller 1976, p. 135).

Pero entonces enfrentamos dos dificultades: (1) comprender por qué cierto plan de vida es valioso para alguien, y (2) juzgar nosotros mismos ese plan de vida.

En relación con (1), a veces no podemos ni siquiera llegar a comprender por qué cierto plan de vida es valioso para alguien. Y en relación con (2), en ocasiones, aunque hayamos comprendido por qué alguien considera valioso su plan de vida, al evaluarlo nos puede resultar condenable por diversas razones. Por ejemplo, porque nos parezca aborrecible por sí mismo, aunque no nos afecte a nosotros ni a terceros; pero también nos puede parecer condenable porque afecta negativamente (según nosotros) a nuestro propio plan de vida, o al de otros. La evaluación, desde luego, la hacemos de acuerdo con nuestros criterios y estándares. 
Pero si un determinado plan de vida nos parece condenable, entonces enfrentamos las siguientes posibilidades:

a) no hacer nada, aunque ese plan de vida ofenda nuestras convicciones (condenamos, pero toleramos, decidimos no intervenir);

b) realizar acciones (si podemos) para modificar, o para impedir la realización del plan de vida juzgado (condenamos y no toleramos, decidimos intervenir).

Podemos entender el concepto de tolerancia en el sentido en que, por ejemplo, lo ha planteado Fernando Salmerón: "Se dice que una persona realiza un acto de tolerancia cuando, en atención a razones y a pesar de tener competencia para hacerlo, no impide algún acto de otra, cuya ejecución lastima sus propias convicciones" (Salmerón 1998, p. 28).

Regresemos al principio de justicia con base en necesidades mencionado al principio:

Una condición necesaria para que una sociedad sea justa es que establezca los mecanismos que garanticen las condiciones y la distribución de bienes de modo que se satisfagan las necesidades básicas de todos sus miembros.

Ejemplos como el del piromaniaco o el estrangulador parecerían dejar claro que, así formulado, el principio es insuficiente y que por lo menos deberíamos agregar algo del siguiente estilo:

Una condición necesaria para que una sociedad sea justa es que establezca los mecanismos que garanticen las condiciones y la distribución de bienes de modo que se satisfagan las necesidades básicas de todos sus miembros, siempre y cuando los planes de vida para los cuales son básicas esas necesidades sean compatibles con la realización de los planes de vida de los demás miembros de la sociedad.

Que sean compatibles con la realización de los planes de vida de los demás miembros de la sociedad quiere decir que su satisfacción no impida la satisfacción de las necesidades básicas de algún otro miembro de la sociedad (en el presente o en el futuro). Éstas son las necesidades básicas legítimas.

Por consiguiente, el principio de justicia basado en la satisfacción de necesidades debería decir:

Una condición necesaria para que una sociedad sea justa es que establezca los mecanismos que garanticen las condiciones que permitan la satisfacción de las legítimas necesidades básicas de todos sus miembros. 


\section{La justicia en una sociedad democrática multicultural}

La aplicación de este principio exige juzgar la compatibilidad de planes de vida. ¿Cómo hacerlo? En una sociedad culturalmente homogénea, la dificultad, aunque grande, parece más tratable. Consideremos, por ejemplo, la forma en que Miller analiza el caso del piromaniaco:

no diríamos que [el piromaniaco] necesita un abundante surtido de cerillos, acceso a establos, [depósitos de madera] etc., sino que necesita ayuda psiquiátrica. Nuestra razón para decir esto es que no consideramos sus intentos de encender fuegos destructivos como constitutivos de un plan de vida inteligible. Por lo tanto, consideramos que las necesidades del piromaniaco no incluyen las condiciones para realizar su presente "plan de vida", sino las condiciones necesarias para que él pueda comprometerse con otros planes de vida que sí son inteligibles. (Miller 1976, p. 135; las cursivas son mías.)

En una sociedad culturalmente homogénea cabe esperar que, aunque no sería fácil, sí sería tratable una controversia acerca de la inteligibilidad, en el sentido aquí utilizado, de un determinado plan de vida, y también sería posible que los miembros de esa sociedad acordaran los procedimientos y las instituciones adecuadas para dirimir la controversia, las cuales incluirían, como en el ejemplo citado, juzgados e instituciones psiquiátricas.

¿Sería radicalmente distinta la situación en una sociedad multicultural? Mi sugerencia es que no, salvo que se requerirá un ejercicio de tolerancia por parte de los miembros de los diferentes grupos culturales que la constituyen. El meollo del asunto consiste en reconocer que hablar de "inteligibilidad" en el sentido sugerido por Miller equivale a hablar de "aceptabilidad", en el sentido débil de "dejar hacer" o "no impedir". Un plan de vida es inteligible —en este contexto- si además de poderlo expresar claramente, lo podemos aceptar. Pero la aceptación puede darse a pesar de que en algunos sentidos el plan de vida ofenda o lastime nuestras convicciones. Es decir, se trata de un plan de vida que podemos tolerar. El plan de vida del piromaniaco es ininteligible, no lo podemos tolerar.

Así, la cuestión de la compatibilidad de planes de vida en una sociedad multicultural requiere que todos sus miembros, pertenecientes a diferentes tradiciones y a diferentes culturas, realicen un continuo esfuerzo de análisis de otros planes de vida y tengan la disposición a tolerarlos hasta el límite posible para ellos (sobre los límites de la tolerancia véase, por ejemplo, Garzón Valdés 2000, cap. 5).

Pero como con frecuencia habrá disputas acerca de esos límites, es decir, acerca de lo que puede tolerarse, una sociedad democrática multicultural debería diseñar y establecer las instituciones legítimas para dirimir las controversias sobre la aceptabilidad para la sociedad multicultural, no sólo para otra cultura, de planes de vida específicos. Es decir, puesto que es de 
esperarse que constantemente haya planes de vida de ciertos miembros de la sociedad, la satisfacción de cuyas necesidades básicas requiera la realización de acciones que serían en principio consideradas como ofensivas por otros sectores de esa sociedad multicultural, entonces se vuelven necesarias instituciones que faciliten las controversias y los medios para dirimir la aceptabilidad social de las acciones que se derivan del plan de vida en cuestión. Diremos que determinadas acciones o costumbres de un grupo son aceptables socialmente (en el contexto de la sociedad democrática) si, aunque ofendan convicciones de los miembros de otro grupo, no les impiden la satisfacción de las legítimas necesidades básicas de los miembros de este otro grupo.

Tales mecanismos deberán estar orientados hacia la resolución de la controversia por procedimientos legítimos aceptados por los diversos sectores de esa sociedad. La controversia debería clausurarse cuando se llegue a alguna de las siguientes situaciones:

a) Mediante los mecanismos diseñados para tal efecto, las instancias pertinentes concluyen que el plan de vida controvertido no impide la satisfacción de las legítimas necesidades básicas de nadie más. Por ejemplo, si en una sociedad democrática donde conviven musulmanes, cristianos y judíos, la conclusión es que el hecho de que las niñas de familias musulmanas lleven pañoletas a la escuela no impide la satisfacción de las necesidades básicas legítimas de cristianos y de judíos, quienes presentan la queja original deberían aceptar tal conclusión y sus consecuencias.

b) La segunda posibilidad es la opuesta a la anterior, es decir, las instancias pertinentes concluyen que las acciones en cuestión sí impiden la satisfacción de legítimas necesidades básicas de ciertos miembros de la sociedad; digamos, cuando un grupo realiza prácticas racistas o de discriminación étnica en cuestiones de salud o de educación, entonces el Estado debe tomar medidas para evitar la realización de las acciones que impiden la satisfacción de las legítimas necesidades básicas de otros.

\section{Responsabilidades del Estado democrático multicultural}

Aquí es donde el Estado en una sociedad multicultural democrática desempeña un papel crucial, pero donde se requieren instituciones y leyes adicionales a las que serían suficientes en una sociedad culturalmente homogénea.

Además de los mecanismos para la resolución de controversias sobre la aceptabilidad de planes de vida, tendrían que desarrollarse otro tipo de políticas; por ejemplo, en el campo educativo, no sólo sería necesario 
contar con sistemas educativos diferenciados, digamos, por la lengua, sino también promover contenidos educativos comunes a todos los sistemas escolares, como programas que hagan ver que la diversidad cultural es valiosa, pues es una forma de existencia de un país que resulta en un enriquecimiento colectivo. Además de exigir la tolerancia, el sistema educativo nacional debería promover el respeto de todas las formas culturales de vida presentes en el país y, más aún, debería promover actitudes antiexcluyentes, es decir, debería promover la antiintolerancia como parte de las condiciones de convivencia armoniosa entre comunidades provenientes de diferentes culturas.

Así pues, la responsabilidad de un Estado democrático en una sociedad multicultural, es decir, en un país donde la diversidad cultural es constitutiva del mismo, no es la de ser tolerante. Es un error categorial pensar que el Estado democrático en una sociedad multicultural debería ser tolerante frente a las diversas culturas o mores que conviven dentro de su marco, pues el Estado no debe comprometerse con ningún punto de vista cultural particular, con ninguna moral ni con ninguna religión específica. Más bien, además de establecer y vigilar que funcionen correctamente las instancias que garanticen una organización social justa, el Estado democrático tiene las siguientes responsabilidades:

a) promover la tolerancia entre los miembros de los diferentes grupos culturales;

b) combatir la intolerancia;

c) establecer y vigilar el correcto funcionamiento de los mecanismos jurídicos e institucionales apropiados para dirimir las controversias acerca de si determinadas necesidades básicas son legítimas, es decir, si un determinado plan de vida y las necesidades básicas que deben satisfacerse para realizarlo son incompatibles con la realización de otros planes de vida, y si afectan negativamente los bienes comunes (la armonía social, la paz, el ambiente, etcétera).

Esos mecanismos tendrían que ser pactados entre representantes del Estado y los representantes legítimos de los diversos grupos culturales; y en su aplicación y vigilancia deberían participar también los representantes de esos grupos.

\section{Las sociedades democráticas multiculturales justas}

La fórmula general de justicia "a cada quien su parte", cuando se aplica en una sociedad democrática y multicultural, debería entenderse como distribución de beneficios y cargas en función de las necesidades legítimas 
de cada quién: "a cada quién según sus legítimas necesidades básicas". Las necesidades básicas legítimas, hemos dicho, son aquéllas indispensables para la realización del plan de vida de las personas, siempre y cuando ese plan de vida no sea incompatible con los planes de vida de los demás miembros de la sociedad.

La realización de los planes de vida de los individuos donde existen diferentes pueblos, o comunidades significativas que se identifican con un determinado pueblo, requiere la sobrevivencia, el florecimiento y el fortalecimiento de la cultura del pueblo donde ha nacido o se han desarrollado esas personas. Por esto, en las sociedades multiculturales los individuos que pertenecen a diferentes pueblos o culturas tienen el derecho a que su pueblo o cultura sobreviva, florezca y se desarrolle, y tienen el derecho a gozar de las condiciones adecuadas para contribuir a la supervivencia y al fortalecimiento de su propia cultura. ${ }^{3}$

Este derecho, he sugerido, es un asunto de justicia social, pues es una condición necesaria para la satisfacción de las legítimas necesidades básicas de esos individuos, y sólo es justa aquella organización social que garantiza la satisfacción de esas necesidades.

Pero tratándose de un derecho - el derecho a la supervivencia y fortalecimiento de la cultura propia (esté o no reconocido en la legislación de un país) - genera una obligación, a saber, la que Garzón Valdés ha llamado la obligación de dinamización, o de la disposición al cambio: "Los representantes de las comunidades indígenas están éticamente obligados a informar a sus miembros acerca de las consecuencias que trae aparejado el rechazo del principio de homogeneidad, es decir, de rechazar las transformaciones que se requieren para que puedan satisfacer sus necesidades básicas [legítimas]" (cfr. Garzón Valdés 1993c, p. 51). Es decir, se trata de la obligación de realizar en la cultura propia y en los planes de vida que ella permite, las transformaciones que sean necesarias para que los individuos puedan satisfacer sus necesidades básicas de manera compatible con la realización de los planes de vida aceptables en la sociedad más amplia de que se trate (a nivel nacional, o a nivel de uniones de estados, o a nivel internacional).

\footnotetext{
${ }^{3}$ Nótese que me refiero al derecho de los individuos a que sobreviva y florezca $s u$ pueblo. El argumento no sugiere ninguna atribución de necesidades básicas a entidades que no sean seres humanos. Por lo tanto, la tesis central de este trabajo es compatible con los siguientes tres principios que ha defendido Ernesto Garzón Valdés: "1. Rechazo del relativismo cultural como fuente de derechos y deberes que exigen aceptación universal. 2. Aceptación del valor del individuo como agente moral. 3. Negación del carácter sacrosanto de las formas de vida colectivas y, por consiguiente, admisión de la posibilidad de su crítica y superación" (Garzón Valdés 1993c, p. 53). Sin embargo, sería necesario aclarar qué se entiende por "relativismo cultural" y, en todo caso, precisar sus diferencias con un modelo "multiculturalista" útil para establecer normas e instituciones en una sociedad culturalmente plural. He desarrollado estas ideas, clave para el argumento de este trabajo, así como mis coincidencias y diferencias con Ernesto Garzón Valdés, en Olivé 1999. Hago estas aclaraciones en vista de una observación de la dictaminadora de Diánoia.
} 


\section{Conclusión}

Una sociedad multicultural donde sólo se toleran los modos de vida de otros es una sociedad menos justa que otra donde existen instituciones y mecanismos para garantizar las condiciones adecuadas para la realización de los planes de vida de cada quién, por más variados que puedan ser, según la cultura a la que pertenezcan, siempre y cuando no impidan la realización de los planes de vida de otros.

El Estado democrático en una sociedad justa tiene la responsabilidad de establecer los mecanismos que garanticen la satisfacción de las legítimas necesidades básicas de todos sus miembros. En las sociedades democráticas multiculturales esto exige garantizar la sobrevivencia de las culturas a las que pertenecen los individuos, para lo cual puede ser necesario establecer políticas diferenciales en la asignación de recursos a favor de las culturas con más desventajas.

El Estado también está obligado a establecer y cuidar el buen funcionamiento de las instancias de vigilancia para dirimir las controversias que inevitablemente surgirán sobre la legitimidad de los planes de vida y de las actividades de los miembros de las diferentes culturas.

\section{BIBLIOGRAFÍA}

Barry, Brian, 1965, Political Argument, Routledge and K. Paul, Londres.

Garzón Valdés, Ernesto, 1993, Derecho, ética y política, Centro de Estudios Constitucionales, Madrid.

__ 1993a, "No pongas tus manos sucias sobre Mozart'. Algunas consideraciones sobre el concepto de tolerancia”, en Garzón Valdés 1993, pp. 401-415; reimpreso en Garzón Valdés 2000, pp. 181-198.

_ _ 1993b, "Necesidades básicas, deseos legítimos y legitimidad política en la concepción ética de Mario Bunge", en Garzón Valdés 1993, pp. 417-435.

__, 1993c, "El problema ético de las minorías étnicas", en L. Olivé (comp.), Ética y diversidad cultural, Fondo de Cultura Económica/UnAm, México, pp. 31-57.

—_, 2000, Instituciones suicidas. Estudios de ética y política, Paidós/UNAM, México. Kymlicka, Will, 1995, Multicultural Citizenship, Oxford University Press, Oxford. Miller, David, 1976, Social Justice, Oxford University Press, Nueva York. Olivé, León, 1999, Pluralismo y multiculturalismo, Paidós/UnAM, México.

Salmerón, Fernando, 1998, Diversidad cultural y tolerancia, Paidós/unAM, México. Villoro, Luis 1998, Estado plural, pluralidad de culturas, Paidós/UnAM, México.

Recibido el 1 de abril de 2003; revisado el 10 de septiembre de 2003; aceptado el 30 de septiembre de 2003 